\title{
Automatic Aesthetic Photo Composition
}

\author{
Roberto Gallea, Edoardo Ardizzone, and Roberto Pirrone \\ DICGIM - University of Palermo \\ \{roberto.gallea, edoardo.ardizzone, roberto.pirrone\}@unipa.it
}

\begin{abstract}
A proper aesthetic composition of photographic content does result in an actual emotional response from the watcher. In this work we propose a fully automatic computational approach to photo composition. This method takes into account well-known and widely adopted aesthetic guidelines relative to picture content as a mean for guiding an optimization framework. The resulting composition is produced as the optimal combination of cropping and retargeting.

The effectiveness of the results achieved by the method are tested and evaluated with several of experiments.
\end{abstract}

Keywords: photo composition, retargeting, visual saliency.

\section{Introduction}

Aesthetic appeal of visual media and art in general is the main goal sought by every artist. From the other hand, it is not easy to achieve such purpose since what is judged as "artistic" is simply subjective and beauty cannot be defined as a universal concept. However, a field in computer science called Computational Aesthetics has emerged. Works in this area aim to use computer science methods to predict, or convey and evoke, the emotional response to a piece of art, or more in general, to any media [17, [19].

This work reports a method to rearrange pictures content taking into account well-established principles of image composition [12, [9], which are given as cues for likely taking more appreciable pictures. Despite of some of their names, these principles are not "rules" but just guidelines for photographers.

Once a picture has been taken, such cues cannot be directly applied anymore, and just digital image editing can be used to rearrange the elements in the image. The skill and techniques required to the editor are often not mastered by the average user.

Considering such perspective, an automatic composition tool can be designed and developed to operate such rearrangement without requiring any intervention from the user. In order to be able to effectively operate such adjustments, composition principles must be coded in quantitative terms and scores, summarizing the perceived quality of the image. Then, an optimization procedure can be executed to extract the transformation maximizing such scores. 
Similarly to the work published in [14, the quality indicators embedded in our tool are based on the following composition guidelines: rule of thirds, diagonal dominance, visual balance and size region. The operator used to rearrange the picture is the combination of cropping and retargeting. Cropping selects a subframe window to use for the final result, while retargeting is used to vary relative distances between subjects. As a result, the rearranged image is completely defined by a set of four values: top-left vertex of the cropping window along its width and height and the amount of retargeting introduced (i.e. the aspect ratio variation). The tuning of such parameters is realized through a search procedure. The output is given as the optimal parameters set producing the result image exhibiting maximum quality score w.r.t. the used aesthetic guidelines.

The paper is arranged as follows: in Section 2 literature about aesthetic photo composition and image retargeting is provided. In Section 3 the system design and implementation is reported: firstly the composition guidelines used are introduced, showing how they are embedded in the system and how their application affects images. Then, an image pre-processing phase describes the objects and features extraction, which are then used to drive the optimization phase. Then, in Section 4 we report the tests taken to assess our system and discuss the results. Finally, in Section 5. final considerations are given and future directions of research are marked.

\section{Previous Works}

Photo composition concerns the arrangement of objects in the picture. Beside photography, composition is a crucial aspect of any visual artistic work. Plenty of techniques are used by professional photographers in their work [16], and, although there not exist golden standard rules for achieving well composed pictures, the application of some general guidelines is recognized to produce eyepleasing results, if applied correctly. Among these principles we mention the rule of thirds, shapes and lines emphasis, diagonal dominance and visual balance [12.

Relatively few attempts have been done to explicitly produce aesthetic image composition. Many works are related to automatic image cropping, for example 25] is based on visual saliency extracted from low-level features [11] and a face detection system. [8] uses the rule of thirds and fifths to choose the format, view and layout parameters of $3 \mathrm{~d}$ rendered objects. [15] use object balance heuristic to determine pleasant user interface layouts. Zhang et al. 28] uses face detection results in conjunction with 14 templates responding to composition rules to create aesthetically pleasing image crops. Santella et al. 22. create image crops based on user eye gaze measurements instead of using composition guidelines. More recent work from Liu et al. [14] uses a search-based crop-and-retarget approach to maximize some measures related to composition rules. Guo et al. 10. extend Liu et al. works taking into account similarity between original and output image to avoid evident distortions in the result image. Battiato et al. [3] compute aesthetic scores on portrait images by considering faces' aspect ratio, lightning and position. 


\subsection{Image Retargeting}

Retargeting system have the goal to vary the aspect ratio of an image without introducing neither severe distortions nor artifacts. They were originally meant to be applied to change media size in order to fit screens with different resolution. In our context image retargeting is used to move objects in the image to better obey to the considered composition rules.

Constraining the application domain to images, a large variety of methods were introduced. Such approaches can be roughly categorized in two different classes: discrete and continuous models [23. Discrete models make an analysis of the image choosing which pixels to dispose or duplicate. In this category lie cropping and seam carving methods [1, 20, 21 and 4]. Some recent works use patches instead of individual pixels and preserve patches coherence between the source and target images, leveraging the presence of repetitive patterns [5], 24], 22. ShiftMap [18 removes entire objects instead of patches, resulting in lesser discontinuity artifacts respect to pixel-based carving.

Continuous methods [27, 6], [13, [26, [7, use a variational approach to define a non-linear resizing operator which attains some goal, such as preserving salient regions shape or distorting preferentially homogeneous regions. These generally provide better results. However, discarding image regions can be a better choice in certain scenarios.

\section{Optimal Photo Composition System}

\subsection{Photo Composition Guidelines}

In photography, there exists a large variety of strategies and guidelines to shot aesthetically appealing pictures. In this work we consider a subset of them, which are the most known and are present almost in every image.

Rule of Thirds. The principal guideline in photography is the rule of thirds [12, 9. This technique considers to subdivide the image canvas in 9 regions of equal size using two horizontal and two vertical lines, as in Fig. 1(a). The four intersections are called "power points". According to this technique, the main subjects of the picture should lie onto these points. In addition prominent lines should also be very close and aligned to those lines. In Fig. 1(a),(b) examples of such concepts are shown.

Diagonal Dominance. Another classical photography guideline is the use of diagonal lines. They create an emphasis effect and help to fixate the viewers' gaze towards the objects passing along them. Fig. 1(c) shows an example of this concept.

Visual Balance. In every sensory art, balance is a crucial concept in composition. Visually balanced images should present the main subjects distributed evenly around the image center, which acts similarly to a center of "mass" from a visual content perspective. In this context, the mass is considered both as the subjects extension and their saliency. Fig. 1(d) is a clear example of such technique. 


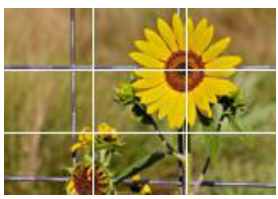

(a)

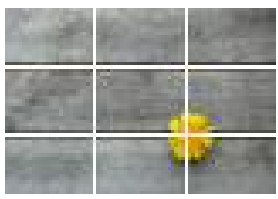

(b)

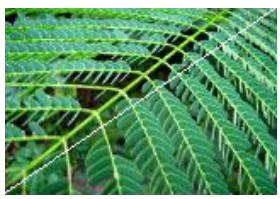

(c)

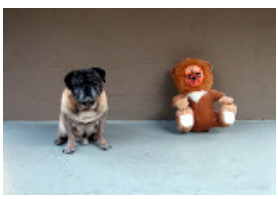

(d)

Fig. 1. Some examples of images exhibiting aesthetic properties such as observation of rule-of-thirds (a) and (b), diagonal dominance (c), visual balance (d)

\subsection{Image Pre-processing}

In order to assign a quality score to the image composition, a proper extraction of the main features of the image has to be accomplished. Such spatial analysis is operated on the main subjects and prominent lines of the image.

Main Subjects and Line Detection. The main subjects are detected by operating a segmentation based on saliency estimation of the image. More specifically, the result of saliency extraction (using Itti's method [11) is thresholded to obtain a subjects map. Then, every remaining object is labeled and information such as area, center of mass and bounding box and are extracted.

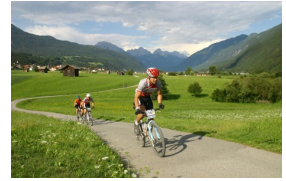

(a)

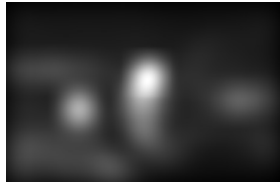

(b)

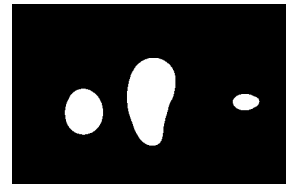

(c)

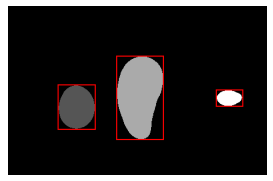

(d)

Fig. 2. Main subject detection chain: (a) input image, (b) saliency detection of the image, (c) saliency thresholding, (d) object labeling and information extraction

Lines are detected in two steps. Firstly, all the pixels belonging to image edges are collected. Then, RANSAC algorithm and least squares are applied to fit the best candidate as a line.

\subsection{Optimization and Output Generation}

Provided that the main subjects and prominent lines have been extracted, the score function representing the aesthetic quality of the image has to be defined. In order to take into account all of the previously described guidelines (rule of thirds, diagonal dominance, and visual balance), the global score function $A_{G}(I)$ needs to be broken into three parts, one for each of the quality criteria as in Equation (1). 


$$
A_{G}(\mathbf{x})=w_{R T} A_{R T}(\mathbf{x})+w_{D D} A_{D D}(\mathbf{x})+w_{V B} A_{V B}(\mathbf{x}),
$$

where $A_{R T}(\mathbf{x})$ is the score related to the Rule of Thirds, $A_{D D}(\mathbf{x})$ is the score related to the diagonal dominance and $A_{V B}(\mathbf{x})$ is the score related to the visual balance quality. The variable vector $\mathbf{x}$ is defined as follows in Equation (2):

$$
\mathbf{x}=\left[x_{t l}, y_{t l}, w, r_{r}\right],
$$

where $x_{t l}$ and $y_{t l}$ are the coordinate of the top left cropping vertex, $w$ is the cropping width and $r_{r}$ is the retargeting ratio.

In the following paragraphs the formulation of each guideline score is described.

Rule of Thirds Score. The score assigned to the rule of thirds fulfillment is composed by two parts, see Eq.(3),

$$
A_{R T}=1 / 3 A_{L D}+2 / 3 A_{O D},
$$

where $A_{L D}$ is function of the distance of prominent lines from third lines and $A_{O D}$ is function of the distance of main objects centers from power points. In detail such, functionals are defined as follows in Eq.(44) and Eq.(5):

$$
\begin{gathered}
A_{L D}=e^{\sum_{i=0}^{N_{L}-1} \min _{j=0}^{3} d\left(l_{i}, l_{t, j}\right)}, \\
A_{P D}=e^{\sum_{i=0}^{N_{O}-1} \min _{j=0}^{3} d\left(o_{i}, p_{t, j}\right)},
\end{gathered}
$$

where $N_{L-1}$ is the number of prominent lines, $l_{i}$ is the $i$-th prominent line, $l_{t, j}$ is the $j$-th third line, $N_{O}$ is the number of main objects, $o_{i}$ is the centroid of the $i$-th main object and $p_{t, j}$ is the $j$-th power points. The function $d(\cdot)$ represents the distance between two points or two lines.

Diagonal Dominance Score. Similarly to the aesthetic score given to distance between prominent lines and third lines, a score for diagonal dominance is assigned according to the distance between diagonal lines and main diagonals of the image. The score is produced as specified in Eq.(6):

$$
A_{D D}=e^{\sum_{i=0}^{N_{D L}-1} \min _{j=0}^{1} d\left(l_{i}, l_{d, j}\right)},
$$

where $N_{D L}$ is the number of diagonal lines in the image, $l_{i}$ is the $i$-th approximatively diagonal lines and $l_{d, j}$ is the $j$-th main diagonal of the image.

Visual Balance Score. The last score used is related to Visual Balance, by assuring that the main objects of the image are evenly distributed across the 
image. The score is assigned by measuring the mass distribution around the center of the image. By relating the mass of an object with its saliency mask extent, a global center of mass of the objects in the scene can be obtained. If such point is close to the image center the composition is considered well-balanced. The score measure is provided in Eq.(7).

$$
A_{V B}=e^{d\left(c_{I}, c_{M}\right)},
$$

where $c_{I}$ is the center of the image and $c_{M}$ is the center of mass of the scene which is calculated according to Eq.(8) :

$$
c_{M}=\frac{\sum_{i=0}^{N_{O}-1} c_{i} m_{i}}{\sum_{i=0}^{N_{O}-1} m_{i}},
$$

where $N_{O}$ is the number of main object in the scene and $c_{i}$ and $m_{i}$ are respectively the center and the extent (in pixel) of the $i$-th object.

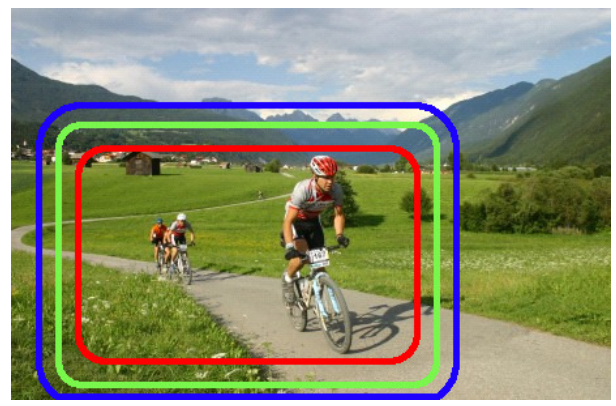

Fig. 3. Example showing that of $E_{G}$ score is not enough restrictive. All the cropping frames shown result in the same aesthetic score value, being the main objects placed across the power points and being equally balanced around the image center.

Main Object Size. Using just the previous scores turns out to be not enough restrictive. Indeed, there are always more than one variables combinations which produce the same aesthetic score. For an example, refer to Fig. 3. Each frame delimits a candidate for an aesthetic composition. The only difference between them is the size of the main object. As demonstrated in [14, salient regions surface area in aesthetic images is a fraction of total image area corresponding, in average, to one of the ratios $r_{1}=0.1, r_{2}=0.56, r_{3}=0.82$. For this reason an additional score is introduced in the scheme as follows 9 .

$$
A_{R S}=\sum_{i=0}^{N_{O}} \max _{j=1,2,3} e^{\frac{\left(r\left(S_{i}\right)-r_{j}\right)^{2}}{2 \tau_{j}}},
$$




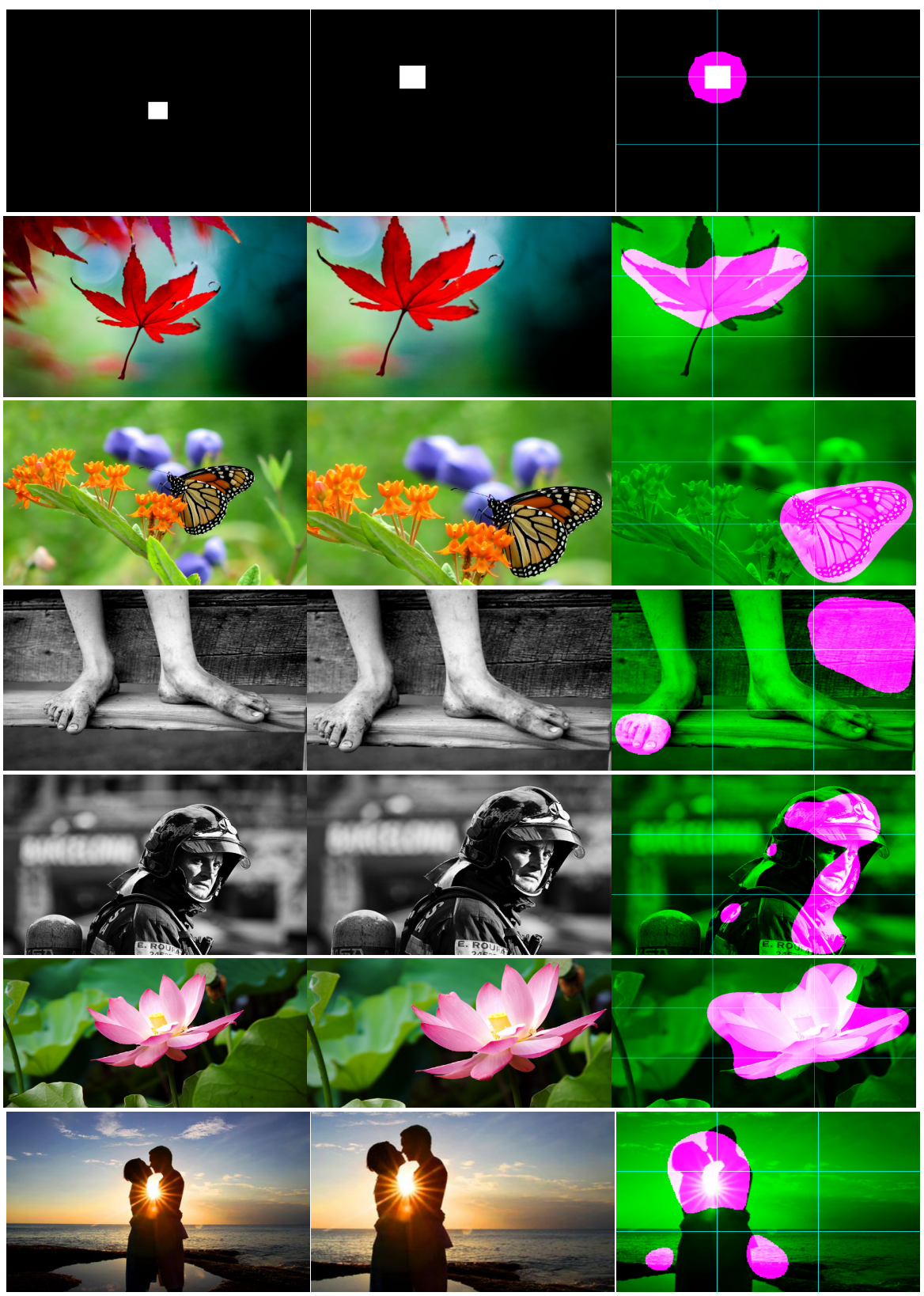

(a) Original image

(b) Aesth. Rearrangement

(c) Saliency and thirds

Fig. 4. Results obtained applying the proposed aesthetic photo composition scheme. (a) original image, (b) output of the aesthetic rearrangement, (c) blending of output image with the detected saliency mask. Thirds lines are overlaid to the images. First row shows a toy example to better illustrate the translate and zoom (cropping) transformation, operated to improve the aesthetic score measure. 
where $r\left(S_{i}\right)$ is the are of the $i$-th object and the values $\tau_{1}=0.07, \tau_{2}=0.3, \tau_{3}=$ 0.16 are recovered fitting a mixture of Gaussians to the histogram of the image dataset used to evaluate most frequent aesthetic object ratio (refer to [14 for details).

According to these additional measure, the final score used for the aesthetic composition becomes as shown in Eq.(10):

$$
A=\left(1-w_{R S}\right) A_{G}+w_{R S} A_{R S},
$$

where the weighting factor $w_{R S}=0.13$ was chosen empirically and used in the rest of the experimental results.

\subsection{Optimization}

The cropping parameters are searched in a 3D space and a fourth parameter manages the retargeting ratio operated using the method described in [7. The optimization approach was chosen between two method: a gradient-descent based method and genetic algorithm. Although the first one is generally faster, the latter provides better results, so it is used for the experiments.

\section{Experimental Results and Discussion}

The approach was applied to several images, some of them are shown in Fig. 4 A preliminary result on a toy test case is shown in the first row of Fig. 4 to better illustrate what are the operations carried out by the system. In this example, the main pattern is translated and zoomed in order to satisfy the Rule of Thirds and Main Object Size criteria. Other results show how in the output images different criteria are predominant. For example rule of thirds is taken into account almost in every case (except for row 4, where visual balance is dominant). Lines play a crucial role in the example in the fourth and the seventh rows, where they get aligned to thirds line. Note that, in fourth rows, visual balance is achieved even if saliency detection is partially wrong. Rule of thirds cannot be fully satisfied due to the position of the detected main objects in the input image, which are very near to the image periphery. This last case is an example of the main limitations of the method, which relies on the saliency detector. Whenever a wrong result is provided during object segmentation phase, the whole processing chain can be partially or totally compromised.

\section{Conclusion and Future Works}

In this paper an approach for automatic aesthetic photo composition was presented. The method embeds photography guidelines (rules of thirds, diagonal dominance and visual balance), to assign a quality measure of an image. Minimization of such score is performed using a crop and retarget transformation. As a result, by following such rules, the output images are more aesthetically 
appealing than the original versions. The method was analytically described and demonstrated with a number of test cases.

Future works would be to embed more well-established photography guidelines and implement the method on mobile systems or in camera firmware, allowing users to take appealing pictures more easily.

Additional attention should be given to the choice of the saliency method, as other top-down based approaches could provide better results.

\section{References}

1. Avidan, S., Shamir, A.: Seam carving for content-aware image resizing. In: ACM SIGGRAPH 2007 Papers. SIGGRAPH 2007. ACM, New York (2007)

2. Barnes, C., Shechtman, E., Finkelstein, A., Goldman, D.B.: PatchMatch: A randomized correspondence algorithm for structural image editing. ACM Transactions on Graphics (Proc. SIGGRAPH) 28(3) (2009)

3. Battiato, S., Moltisanti, M., Ravì, F., Bruna, A.R., Naccari, F.: Aesthetic scoring of digital portraits for consumer applications, pp. 866008-866008-10 (2013), http://dx.doi.org/10.1117/12.2005211

4. Battiato, S., Farinella, G.M., Puglisi, G., Ravì, D.: Content-aware image resizing with seam selection based on gradient vector flow. In: ICIP, pp. 2117-2120 (2012)

5. Cho, T.S., Butman, M., Avidan, S., Freeman, W.T.: The patch transform and its applications to image editing. In: CVPR (2008)

6. Gal, R., Sorkine, O., Cohen-Or, D.: Feature-aware texturing. In: Proceedings of Eurographics Symposium on Rendering, pp. 297-303 (2006)

7. Gallea, R., Ardizzone, E., Pirrone, R.: Real-time content-aware image resizing using reduced linear model. In: ICIP 2010, IEEE International Conference on Image Processing. IEEE Computer Society, Washington, DC (2010)

8. Gooch, B., Reinhard, E., Moulding, C., Shirley, P.: Artistic composition for image creation. In: Proceedings of the 12th Eurographics Workshop on Rendering Techniques, pp. 83-88. Springer, London (2001)

9. Grill, T., Scanlon, M.: Photographic Composition: Guidelines for Total Image Control Through Effective Design. Watson-Guptill Publications (1983)

10. Guo, Y.W., Liu, M., Gu, T.T., Wang, W.P.: Improving photo composition elegantly: Considering image similarity during composition optimization. Comp. Graph. Forum 31(7 pt. 2), 2193-2202 (2012)

11. Itti, L., Koch, C., Niebur, E.: A model of saliency-based visual attention for rapid scene analysis. IEEE Trans. Pattern Anal. Mach. Intell. 20(11), 1254-1259 (1998)

12. Krages, B.: Photography: The Art of Composition. Allworth Press (2005)

13. Krähenbühl, P., Lang, M., Hornung, A., Gross, M.: A system for retargeting of streaming video. In: ACM SIGGRAPH Asia 2009 Papers, SIGGRAPH Asia 2009, pp.126:1-126:10. ACM, New York (2009)

14. Liu, L., Chen, R., Wolf, L., Cohen-Or, D.: Optimizing photo composition. Computer Graphic Forum (Proceedings of Eurographics) 29(2), 469-478 (2010)

15. Lok, S., Feiner, S., Ngai, G.: Evaluation of visual balance for automated layout. In: Proceedings of the 9th International Conference on Intelligent User Interfaces, UI 2004, pp. 101-108. ACM Press, New York (2004)

16. Martinez, B., Block, J.: Visual forces: an introduction to design. Prentice Hall (1988) 
17. Peters, G.: Aesthetic primitives of images for visualization. In: 11th Int'l Conference on Information Visualization 2007, pp. 316-325 (July 2007)

18. Pritch, Y., Kav-Venaki, E., Peleg, S.: Shift-map image editing. In: ICCV 2009, Kyoto, pp. 151-158 (September 2009)

19. Rivotti, V., Proenì, J., Jorge, J.A., Sousa, M.C.: Composition principles for quality depiction and aesthetics. In: Cunningham, D.W., Meyer, G.W., Neumann, L., Dunning, A., Paricio, R. (eds.) Computational Aesthetics: Eurographics Workshop on Computational Aesthetics in Graphics, Visualization and Imaging, Banff, Alberta, Canada, June 20-22, pp. 37-44. Eurographics Association (2007)

20. Rubinstein, M., Shamir, A., Avidan, S.: Improved seam carving for video retargeting. ACM Transactions on Graphics (SIGGRAPH) 27(3), 1-9 (2008)

21. Rubinstein, M., Shamir, A., Avidan, S.: Multi-operator media retargeting. ACM Transactions on Graphics (Proceedings SIGGRAPH 2009) 28(3), 1-11 (2009)

22. Santella, A., Agrawala, M., DeCarlo, D., Salesin, D., Cohen, M.: Gaze-based interaction for semi-automatic photo cropping. In: Proceedings of the SIGCHI Conference on Human Factors in Computing Systems, CHI 2006, pp. 771-780. ACM, New York (2006)

23. Shamir, A., Sorkine, O.: Visual media retargeting. In: ACM SIGGRAPH ASIA 2009 Courses, SIGGRAPH ASIA 2009, pp. 11:1-11:13. ACM, New York (2009)

24. Simakov, D., Caspi, Y., Shechtman, E., Irani, M.: Summarizing visual data using bidirectional similarity. In: CVPR (2008)

25. Suh, B., Ling, H., Bederson, B.B., Jacobs, D.W.: Automatic thumbnail cropping and its effectiveness. In: Proceedings of the 16th Annual ACM Symposium on User Interface Software and Technology, UIST 2003, pp. 95-104. ACM, New York (2003)

26. Wang, Y.S., Lin, H.C., Sorkine, O., Lee, T.Y.: Motion-based video retargeting with optimized crop-and-warp. ACM Trans. Graph. 90, 90:1-90:9 (2010)

27. Wang, Y.S., Tai, C.L., Sorkine, O., Lee, T.Y.: Optimized scale-and-stretch for image resizing. In: ACM SIGGRAPH Asia 2008 Papers, SIGGRAPH Asia 2008, pp. 118:1-118:8. ACM, New York (2008)

28. Zhang, M.: W.: Auto cropping for digital photographs. In: IEEE Conf. on Multimedia and Expo (2005) 